\title{
Activation of nicotinamide N-methyltrasferase and increased formation of 1-methylnicotinamide (MNA) in atherosclerosis
}

\author{
Łukasz Mateuszuk ${ }^{1}$, Tamara I. Khomich², Ewa Słomińska ${ }^{3}$, Mariusz Gajda ${ }^{4}$, \\ Luiza Wójcik $^{5}$, Magdalena Łomnicka ${ }^{1}$, Pawel Gwóźdź ${ }^{1}$, Stefan Chłopicki ${ }^{1}$ \\ ${ }^{1}$ Department of Experimental Pharmacology, Chair of Pharmacology, Jagiellonian University, Medical College, \\ Grzegórzecka 16, PL 31-531 Kraków, Poland \\ ${ }^{2}$ Institute of Pharmacology and Biochemistry, NAS of Belarus, Grodno, Belarus \\ ${ }^{3}$ Department of Biochemistry, Medical Academy, Gdańsk, Poland \\ ${ }^{4}$ Chair of Histology, Jagiellonian University, Kraków, Poland \\ ${ }^{5}$ NeuroRepair Department, Medical Research Centre Polish Academy of Sciences, Warszawa, Poland
}

Correspondence: Stefan Chłopicki, e-mail: s.chlopicki@jmrc.org.pl

\begin{abstract}
:
Nicotinamide N-methyltrasferase (NMMT) catalyzes the conversion of nicotinamide (NA) to 1-methylnicotinamide (MNA). Recent studies have reported that exogenous MNA exerts anti-thrombotic and anti-inflammatory activity, suggesting that endogenous NMMT-derived MNA may play a biological role in the cardiovascular system.

In the present study, we assayed changes in hepatic NNMT activity and MNA plasma levels along the progression of atherosclerosis in apoE/LDLR ${ }^{-/}$mice, as compared to age-matched wild-type mice. Atherosclerosis progression in apoE/LDLR ${ }^{-/-}$mice was quantified in aortic root, while hepatic NNMT activity and MNA plasma concentrations were concomitantly measured in 2-, 3-, 4-, and 6-month-old mice.

In apoE/LDLR ${ }^{-/}$mice, atherosclerotic plaques developed in the aortic roots beginning at the age of 3 months and gradually increased in size, macrophage content, and inflammation intensity over time, as detected by Oil-Red O staining, CD68 immunostaining, and in situ zymography (MMP2/MMP9 activity). Hepatic NNMT activity was upregulated approximately two-fold in apoE/LDLR ${ }^{-/}$mice by the age of 2 months, as compared to wild-type mice $(1.03 \pm 0.14 \mathrm{vs} .0 .64 \pm 0.23 \mathrm{pmol} / \mathrm{min} / \mathrm{mg}$, respectively). MNA plasma concentrations were also elevated approximately two-fold $(0.30 \pm 0.13 v s .0 .17 \pm 0.04 \mu \mathrm{mol} / 1$, respectively). As atherosclerosis progressed, hepatic NMMT activity and MNA plasma concentrations increased five-fold in 6-month-old apoE/LDLR ${ }^{-/-}$ mice at the stage of advanced atherosclerotic plaques (NMMT activity: $2.29 \pm 0.34 \mathrm{pmol} / \mathrm{min} / \mathrm{mg}$, MNA concentration: $1.083 \pm$ $0.33 \mu \mathrm{mol} / \mathrm{l})$.

In summary, the present study demonstrated that the progression of vascular inflammation and atherosclerosis was associated with the upregulation of hepatic NNMT activity and subsequent increase in endogenous MNA plasma levels. Given the anti-thrombotic and anti-inflammatory properties of exogenous MNA, robust activation of an endogenous NA-MNA pathway in atherosclerosis may play an important compensatory role.
\end{abstract}

Key words: nicotinamide N-methyltransferase, 1-methylnicotinamide, atherosclerosis, vascular inflammation, ApoE/LDLR ${ }^{-/-}$mice 
Abbreviations: Met-2PY - 1-methyl-2-pyridone-5-carboxamide, Met-4PY - 1-methyl-4-pyridone-5-carboxamide, MNA 1-methylnicotinamide, NA - nicotinamide, NNMT - nicotinamide $\mathrm{N}$-methyltransferase

\section{Introduction}

Nicotinamide N-methyltrasferase (NNMT) is a cytosolic enzyme that catalyzes the transfer of methyl group of S-adenosylmethionine (SAM) to nicotinamide (NA) producing 1-methylnicotinamide (MNA) and S-adenosylhomocysteine (SAH) [3, 33]. NNMT activity is localized predominantly in the mammalian liver, but it can also be detected in the heart, placenta, lung, brain, adrenals, skeletal muscles, kidneys [3, 39], adipose tissue [32], and tumor cells [37]. NNMT also plays a role in the biotransformation of drugs, which undergo methylation via methyltransferases $[26,45]$, but the physiological role of this enzyme appears to be linked to the activity of its major substrate - NA (niacin, vitamin B3), in addition to its major product - MNA. NA is a water-soluble vitamin essential for energy supply, cell viability, and resistance to stress or injury [28]. Moreover, it has recently become apparent that MNA, long considered an inactive metabolite of NA, possess anti-thrombotic [10], antiinflammatory [8], gastroprotective [9], and vasoprotective [5] properties.

Interestingly, it has been reported that NNMT activity can be markedly increased in a range of different circumstances, for example, in rats treated with ligands for peroxisome proliferator activated receptor $\alpha(\operatorname{PPAR} \alpha)[14]$; in mice treated with alkylating agents [30] or with phenobarbital [16]; in hepatoma cells with expression of the hepatitis $\mathrm{C}$ virus core protein [27]; in mouse models of bacteria-induced fulminant hepatitis [15]; and in patients with liver cirrhosis $[13,31]$ or Parkinson's disease [4, 29]. Moreover, heightened NNMT activity was reported in many types of cancer cells, including papillary thyroid cancer cells [47], renal carcinoma cells [49], human stomach adenocarcinoma cells [23], oral squamous carcinoma cells [38], colorectal cancer cells [35], and radio-resistant bladder cancer cells [24]. The upregulation of NMMT in cancer cells suggests a possible role for NMMT in tumor growth, migration, and metastasis $[38,46]$. Indeed, it was hypothesized that NNMT activity in cancer cells or NNMT concentra- tions in serum may provide a useful biomarker in patients with cancer [35, 37].

The NNMT gene has been cloned in both humans and mice $[2,48]$. However, the biological significance of alterations in NNMT activity in various pathological conditions and the factors regulating NNMT expression remain largely unknown. It was recently demonstrated that NNMT gene expression in cancer cells may be regulated by the hepatocyte nuclear factor $\beta$ (HNF-1 $\beta$ ) binding to specific HNF-1 sites in the NNMT basal promoter region [47], as well as by signal transducers and activators of transcription 3 (STAT3) [42]. It was also suggested that NNMT activity is determined by genetic polymorphisms. In contrast to others [6], some authors [41] have demonstrated that NNMT's genetic polymorphisms influence the concentration of homocysteine in plasma. Furthermore, children with NNMT polymorphism, who have been exposed to medicines and/or a low nicotinamide diet, have an eight-fold increased risk of developing congenital heart defects [44].

Finally, NNMT activity may be involved in regulating the biological activity of endogenous MNA. Recently, we suggested that MNA formed in the liver by nicotinamide $\mathrm{N}$-methyltrasferase can be an endogenous activator of the COX-2/PGI 2 pathway and may play an important regulatory role in limiting thrombosis and inflammatory processes in the cardiovascular system [10]. The formation and progression of atherosclerotic plaques are driven by vascular inflammation and thrombosis, and both of these processes may be associated with changes in NNMTderived MNA formation. We tested this hypothesis in the present study and investigated whether hepatic NNMT activity and endogenous MNA plasma concentrations are altered along the progression of atherosclerosis in apoE/LDLR ${ }^{-/}$mice.

\section{Materials and Methods}

\section{Animals}

ApoE/LDLR ${ }^{--}$mice on a $\mathrm{C} 57 \mathrm{BL} / 6 \mathrm{~J}$ genetic background (obtained from The Jackson Laboratory, Maine, USA) and wild-type C57BL/6J mice were used in the study. 
Mice were maintained on 12-h dark/12-h light cycles in air-conditioned rooms $\left(22.5 \pm 0.5^{\circ} \mathrm{C}, 50 \pm 5 \%\right.$ humidity) with access to standard chow diet and water ad libitum. All animal care procedures conformed to the Guide for the Care and Use of Laboratory Animals published by the US National Institutes of Health (NIH Publication No. 85-23, revised 1996) and were approved by the Jagiellonian University Ethics Committee on Animal Experiments.

ApoE/LDLR ${ }^{-/}$and C57BL/6J mice at the age of 2, 3, 4, and 6 months $(\mathrm{n}=6)$ were injected with $1000 \mathrm{IU}$ of fraxiparine (Sanofi-Synthelabo, Santea, France) and anesthetized $(10 \mathrm{mg} / \mathrm{kg}$ of thiopental ip Sandoz, Vienna, Austria). The entire heart was dissected, embedded in OCT-compound (Jung, Germany) and snap-frozen at $-80^{\circ} \mathrm{C}$ to examine atherosclerosis progression. A liver sample (approximately $300 \mathrm{mg}$ ) was taken and washed with cold $0.9 \%$ (w/v) sodium chloride immediately after isolation to assess hepatic NMMT activity. To analyze the concentration of MNA and its metabolites in plasma, blood samples were taken from the left ventricle of the heart and centrifuged for $6 \mathrm{~min}(14,000 \times \mathrm{g})$ to isolate plasma.

\section{Quantification of atherosclerosis (lipid deposi- tion, macrophage contents, and in situ zymo- graphy)}

Ten micrometer-thick sections of the aortic root were cut on a Leica CM1800 cryostat according to the standardized cross-section protocol previously described in detail [24].

In short, serial sections from the proximal $1 \mathrm{~mm}$ of the aortic root were collected on separate slides, air dried, and fixed in 4\% phosphate-buffered paraformaldehyde (oil red) or left unfixed and stored at $-20^{\circ} \mathrm{C}$ (zymography/immunohistochemistry).

To assess lipid deposition, sections were stained with Oil Red-O (ORO) and examined under an Olympus BX50 microscope (Olympus, Tokyo, Japan). Images of the aortic roots were obtained using an Olympus DP71 digital CCD camera. The total lesion area, as revealed by ORO staining, was measured semiautomatically for each slide using the Zeiss LSM Image Browser 3.2 software (Carl Zeiss, Jena, Germany). The mean lesion area was calculated using a minimum of eight slices per animal.

To demonstrate [34] gelatinase activity in the zymography assay, non-fixed aorta sections were thawed and incubated for $2 \mathrm{~h}$ at $37^{\circ} \mathrm{C}$ in a dark humid cham- ber with a reaction buffer containing $50 \mathrm{mg} / \mathrm{ml}$ of FITC-labeled DQ-gelatin (Molecular Probes, Eugene, OR, USA). Sections were rinsed in PBS, fixed in cold $4 \%$ paraformaldehyde for $15 \mathrm{~min}$, placed on the mounting medium (Dako, Glostrup, Denmark) and observed using fluorescence microscopy. The peptide fluorescence released from FITC-gelatin is representative of local tissue gelatinase activity. Carl Zeiss AxioVision 4.6 software was used to measure the fluorescence intensity, which was measured in at least 3 sections from each aortic root sample.

For the indirect immunohistochemistry of macrophages, sections previously used for zymography were rinsed in PBS and preincubated with 5\% normal goat serum with $2 \%$ dry milk. The sections were then incubated overnight with rat anti-mouse CD68 (Serotec, Oxford, UK; \# MCA1957XZ; diluted 1:800), and then Cy3-conjugated goat anti-rat antiserum (Jackson IR, West Grove, PA) was applied. Sections were examined using an epifluorescence Olympus BX50 microscope equipped with appropriate filter cubes to show Cy3 (red) and FITC (green) fluorescence.

\section{Determination of NNMT activity in the liver}

To measure the activity of N-methyltransferase, the fluorometric method of Sano et al. [36] was used with a slight modification. The method was based on the enzyme reaction at $\mathrm{pH} 8.6$ using 4-methylnicotinamide (4-MNA) as the methyl acceptor substrate in the presence of cofactor, S-adenosyl-L-methionine (SAM), and the fluorometric determination of the product, 1,4-dimethylnicotinamide, by means of its reaction with 4-methoxybenzaldehyde. The livers of mice were excised and washed with cold $0.9 \%$ (w/v) sodium chloride, immediately frozen in liquid nitrogen, and stored at $-80^{\circ} \mathrm{C}$. The tissue was homogenized 1:9 (w/v) in cold PBS buffer $(\mathrm{pH} 7.4)$ with a Teflon-glass homogenizer. The homogenate was centrifuged at $12,000 \times \mathrm{g}$ for $20 \mathrm{~min}$ at $4^{\circ} \mathrm{C}$. The supernatant was used immediately to assay enzymatic activity. Protein concentration was determined using BSA as the standard. The reaction mixture consisted of $12 \mu \mathrm{l}$ of $2 \mathrm{mM}$ dithiothreitol, $12 \mu \mathrm{l}$ of $0.8 \mathrm{M}$ Tris- $\mathrm{HCl}$ buffer ( $\mathrm{pH} 8.6$ ), $25 \mu \mathrm{l}$ of $16 \mathrm{mM}$ 4-MNA, $50 \mu \mathrm{l}$ of $0.4 \mathrm{mM}$ SAM in $0.1 \mathrm{mM}$ of sulfuric acid and $100 \mu \mathrm{l}$ of enzyme preparation. The mixture was incubated at $37^{\circ} \mathrm{C}$ for $80 \mathrm{~min}$. Substrate N-methylation proceeded linearly during the incubation period. The enzyme reaction was terminated by heating in boiling 
water for $2 \mathrm{~min}$. The same procedure was used to prepare the blank; however, the reaction was stopped immediately without incubation. These reaction mixtures were centrifuged at $10,000 \times \mathrm{g}$ for $5 \mathrm{~min}$ at room temperature. A $100 \mu \mathrm{l}$ aliquot of the supernatant was poured into a $1.5-\mathrm{ml}$ stopper test tube with the addition of $1 \mathrm{ml}$ of $0.02 \mathrm{M}$ 4-methoxybenzaldehyde in $35 \%(\mathrm{v} / \mathrm{v})$ aqueous 2-methoxyethanol and $100 \mu \mathrm{l}$ of $0.5 \mathrm{M}$ aqueous sodium hydroxide. The tube was heated in a water bath $\left(100^{\circ} \mathrm{C}\right)$ for $15 \mathrm{~min}$. After cooling, the fluorescence intensity was measured using a Perkin Elmer LS50B spectrofluorometer with excitation at $418 \mathrm{~nm}$ and emission at $490 \mathrm{~nm}$.

The quantity of the reaction product obtained, 1,4DMN, was calculated by the calibrating curve for 1,4-DMN at the concentration range of $0.2-2.0 \mathrm{nM}$. At this range of concentration, the calibrating curve was linear. The activity of N-methyltransferase was expressed in $\mathrm{pmol} / \mathrm{min} / \mathrm{mg}$ of protein.

\section{Determination of the concentration of MNA and its metabolites in plasma}

The concentrations of endogenous MNA and its metabolites in plasma samples were measured using liquid chromatography mass spectrometry (LC/MS), as described by Słomińska et al. [41]. Chromatographic separation was performed using a $3 \mu \mathrm{m}$ Hypersil C18-BDS $150 \times 2.0 \mathrm{~mm}$ column. The mobile phase A consisted of $10 \mathrm{mM}$ nonafluoropentanoic acid (NFPA) in water, while mobile phase B consisted of $100 \%$ acetonitrile. The mobile phase was delivered at 0.2 $\mathrm{ml} / \mathrm{min}$ in a gradient from $0 \%$ to $60 \% \mathrm{~B}$ in $12 \mathrm{~min}$. The mass detector (Thermo-Finnigan LCQ Advantage, Waltham, MA, USA), which contains an electrospray ion source (ESI), was set to a positive single ion monitoring (SIM) mode to detect $[\mathrm{M}+\mathrm{H}]^{+}$species of NA, MNA, Met-2PY, Met-4PY and a collision energy set at $25 \%$. The internal standard (2-chloroadenosine) signal was extracted from full MS mode. The electrospray cone voltage was set at $4.5 \mathrm{kV}$, while the heated capillary temperature was set at $275^{\circ} \mathrm{C}$. Sheath gas flow was set at 35 arbitrary units. Ion optic parameters were optimized using standard instrument procedures during the nicotinamide infusion. Mouse plasma was deproteinized using $10 \%$ trichloroacetic acid, which was subsequently extracted using diethyl ether. There was a $75-95 \%$ recovery of Met-2PY, Met-4PY, and NA, which was added to the samples.
The coefficient of variation was below $10 \%$ for repeated injections on the same day [40].

\section{Statistical analysis}

Results are expressed as the means $\pm \mathrm{SD}$. Comparison of the means was assessed by ANOVA and post hoc Scheffe's tests. The non-parametric Mann-Whitney test was used for the analysis of data regarding the quantification of atherosclerosis, NNMT activity, and metabolite concentrations. Differences between means were considered significant at $\mathrm{p} \leq 0.05$. Statistical analysis was performed using Prism 5 ver. 5.01 (GraphPad Software Inc.).

\section{Results}

\section{Progression of atherosclerosis and vascular in- flammation}

Atherosclerotic lesions were not detected in the aortic roots of 2-month-old apoE/LDLR ${ }^{-/}$mice, as evidenced by the lack of Oil-Red O staining, CD68 immunostaining, and negligible gelatinase activity in in situ zymography (MMP-2/MMP-9) (Fig. 1). However, in the intima of the aortic roots in 3-month-old apoE/LDLR ${ }^{-/}$mice, lipid deposition, macrophage accumulation, and increased gelatinase activity were easily detected. In 4-month-old apoE/LDLR ${ }^{-/-}$mice, atherosclerotic plaques in the aortic roots were considerable in size (Oil-Red O; $3.35 \pm 1.41 \mu \mathrm{m}^{2} \times 10^{5}$ ) and continued to increase in the later stages of plaque development in 6-month-old mice (Fig. 1). It is interesting to note that $\mathrm{CD}-68$ positive macrophages constituted the major component of the area of atherosclerotic plaques at the early stage of plaque development, while in 4- and 6-month-old apoE/LDLR ${ }^{-/}$ mice, macrophage content comprised a smaller component of the total lesion area stained by Oil-Red O (Fig. 1). Gelatinase activity detected by in situ zymography increased gradually in the aortic roots between 3- and 6-month-old ApoE/LDLR ${ }^{--}$mice (Fig. 1). As shown in Figure 2, gelatinase activity only partially co-localized with CD68 immunostaining. These data suggest that apart from CD68 positive macrophages, other cell types were responsible for increased gelati- 

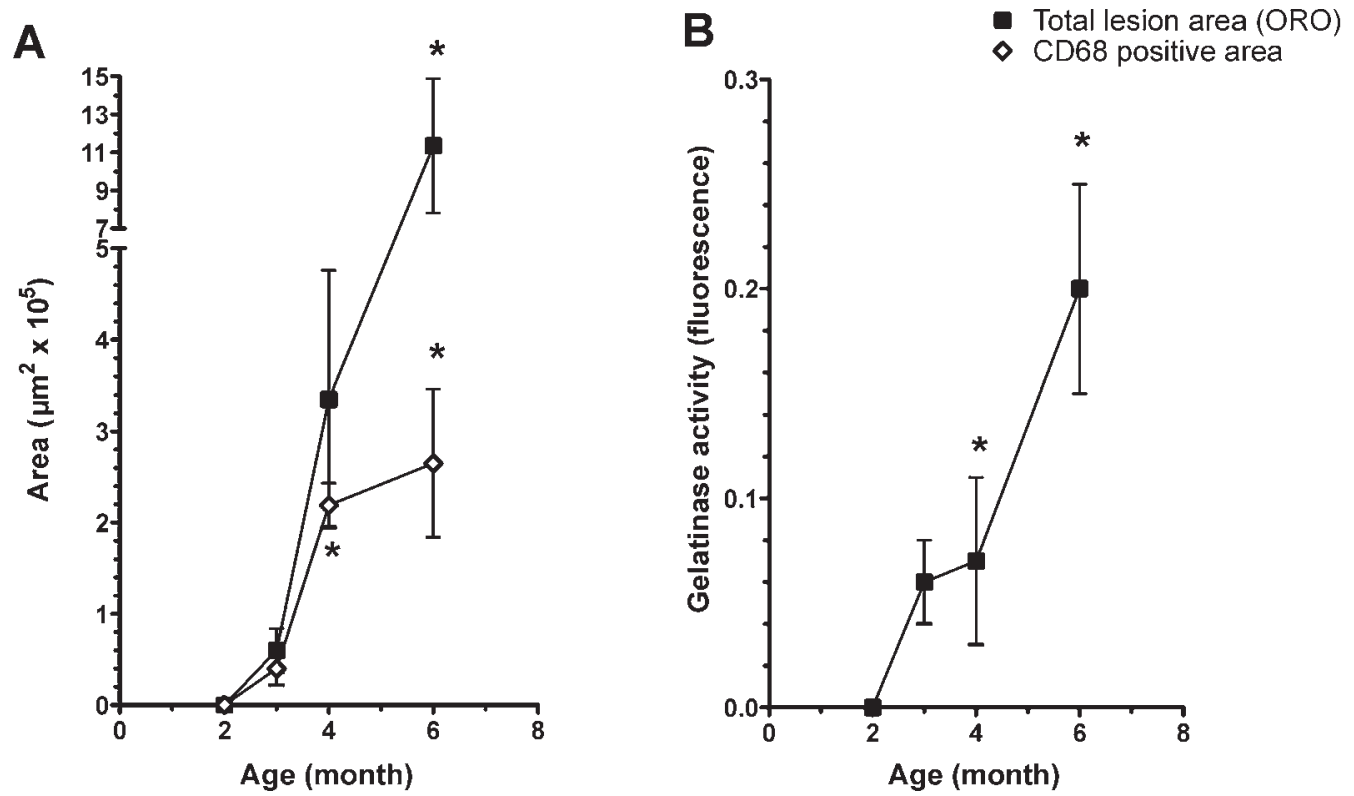

Fig. 1. Progression of atherosclerosis in apoE/LDLR ${ }^{-1-}$ mice assessed on the basis of Oil Red O (ORO) staining (total lesion area), CD $68 \mathrm{immu-}$ nostaining (A), and gelatinase activity (MMP-2 and MMP-9) (B). Results are presented as the mean \pm SD $\left(n=4-5,{ }^{*}\right.$ denotes $p<0.05$ vs. 2-monthold apoE/LDLR ${ }^{-1-}$ mice)
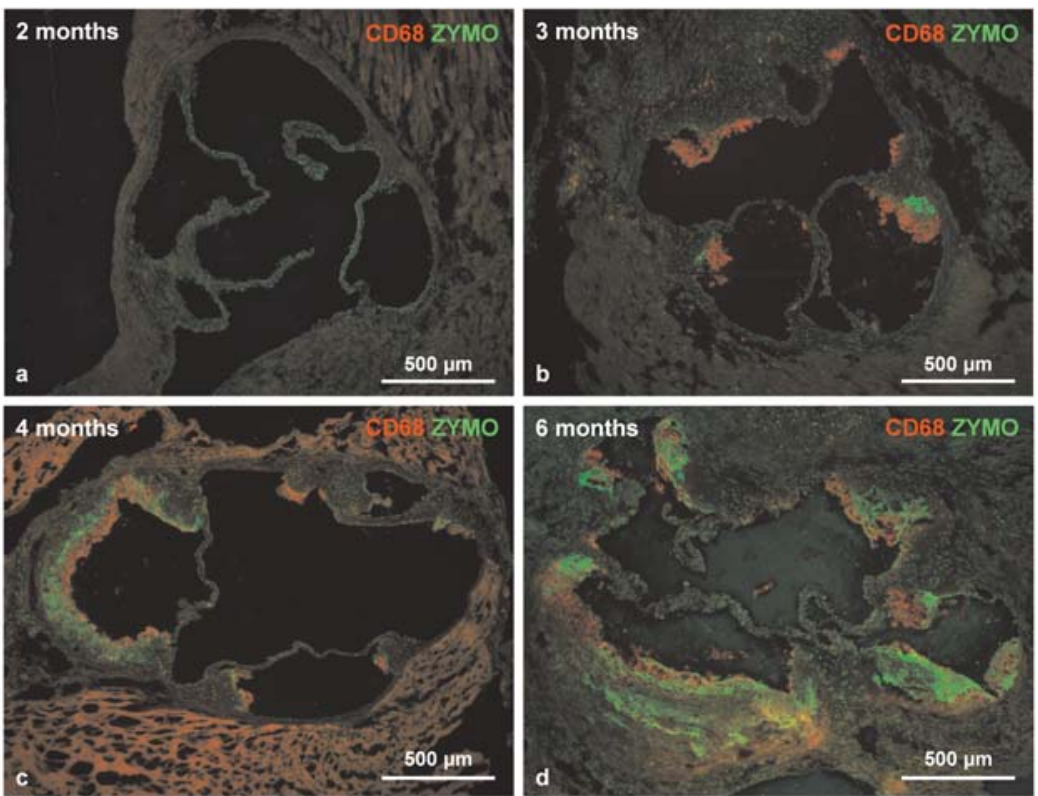

Fig. 2. Combined CD68-immunohistochemistry (red) and gelatinase zymographic staining (green) in the aortic roots of 2-(2a), 3- (2b), 4(2c, e-g) and 6-month-old apoE/LDLR ${ }^{-1-}$ mice. Limited colocalization between CD68-immunoreactive macrophages (2e,f, arrows) and metalloproteinase activity (2 e,g, arrowheads) is evident
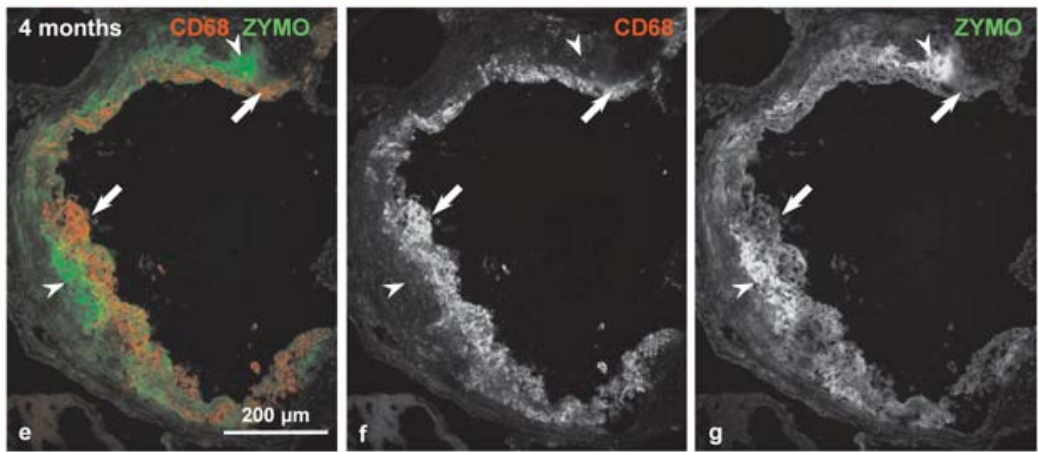


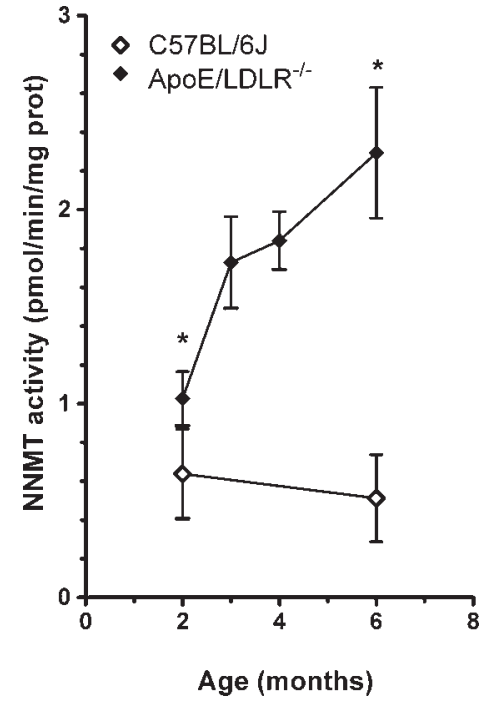

Fig. 3. Increased hepatic NNMT activity along the progression of atherosclerosis in apoE/LDLR ${ }^{-/-}$mice. Results are presented as the mean $\pm \mathrm{SD}\left(\mathrm{n}=4-5,{ }^{*}\right.$ denotes $\mathrm{p}<0.05 \mathrm{vs}$. age-matched C57BL/6J mice)

nase activity within the plaque area, or that gelatinase activity was present extracellularly.

\section{Changes in hepatic NNMT activity}

Hepatic NNMT activity was elevated in 2-month-old apoE/LDLR ${ }^{-1-}$ mice, as compared to age-matched C57BL $/ 6 \mathrm{~J}$ mice $(1.03 \pm 0.14$ vs. $0.64 \pm 0.23 \mathrm{pmol} /$ $\mathrm{min} / \mathrm{mg}$ in apoE/LDLR ${ }^{-/}$and wild-type mice, respectively). Furthermore, in contrast to wild-type C57BL/6J mice, where NNMT activity remained similar in 2-, 3-, 4- and 6-month-old animals, NNMT activity gradually increased with animal age in apoE/LDLR ${ }^{-/}$ mice (Fig. 3). Hepatic NNMT activity was enhanced approximately five-fold in 6-month-old apoE/LDLR ${ }^{-/}$ mice $(2.29 \pm 0.34 \mathrm{pmol} / \mathrm{min} / \mathrm{mg})$, with advanced atherosclerotic plaques.

\section{Changes in the concentration of NA, MNA, Met-2PY and Met-4PY in plasma}

In wild-type mice plasma concentrations of NA, MNA, Met-2PY, and Met-4PY were not significantly altered by age (Fig. 4). Interestingly, plasma concentrations of NA and MNA were elevated approximately two-fold in 2-month-old apoE/LDLR ${ }^{-1-}$ mice, as compared to age-matched $\mathrm{C} 57 \mathrm{BL} / 6 \mathrm{~J}$ control mice $(\mathrm{NA} ; 7.92 \pm$ 2.61 vs. $3.11 \pm 0.80 \mu \mathrm{mol} / 1$, MNA; $0.30 \pm 0.13$ vs.
$0.17 \pm 0.04 \mu \mathrm{mol} / 1$ in apoE/LDLR ${ }^{-/}$and $\mathrm{C} 57 \mathrm{BL} / 6 \mathrm{~J}$ mice, respectively). The elevations in plasma concentrations of NA and MNA were more pronounced in older apoE/LDLR ${ }^{-/}$mice. At the age of 6 months, NA and MNA concentrations were approximately five times greater in comparison with age-matched wildtype mice (NA; $12.46 \pm 1.57 v s .4 .96 \pm 0.52 \mu \mathrm{mol} / 1$, MNA; $1.08 \pm 0.34$ vs. $0.17 \pm 0.04 \mu \mathrm{mol} / 1$ in apoE/LDLR ${ }^{-/-}$and $\mathrm{C} 57 \mathrm{BL} / 6 \mathrm{~J}$ mice, respectively). In addition to the elevated plasma concentrations of NA and MNA, the levels of Met-2PY and Met-4PY in plasma were also significantly elevated in 6-monthold apoE/LDLR ${ }^{-1-}$ mice, as compared to wild-type mice (Fig. 4).

\section{Discussion}

In the present work we demonstrated for the first time, that NMMT activity and endogenous MNA levels were elevated in the early phase of atherosclerotic development and increased gradually with the progression of atherosclerosis in apoE/LDLR ${ }^{-/}$mice.

The progression of atherosclerotic lesions in apoE/LDLR ${ }^{-/}$mice has been well characterized $[12$, 21]. It is known that total- and LDL-cholesterol levels are highly elevated in apoE/LDLR ${ }^{-/}$mice at an early age [21], resulting in the formation of atherosclerotic lesions in the aortic roots and other regions in an agedependent manner. These lesions are characterized by numerous biochemical, structural, and functional changes resembling human pathology, including oxidized lipid accumulation, endothelial dysfunction, and vascular inflammation [20, 21, 23].

In this study, we assessed vascular inflammation in atherosclerotic plaques by CD68 immunostaining, which reflects subendothelial macrophage accumulation. In addition, we assessed the activity of MMP-2 and MMP-9 by in situ zymography via the gelatinase assay. Both MMP-2 and MMP-9 play a major role in degradation of the vascular extracellular matrix (ECM) [17], and their biological activity may be linked to the activation of macrophages, T lymphocytes, smooth muscle cells, and endothelial cells [25]. We found that macrophages constituted the major component of plaque area in the early stages, with other cell types and non-cellular components dominating at later stages. Interestingly, gelatinase activity 

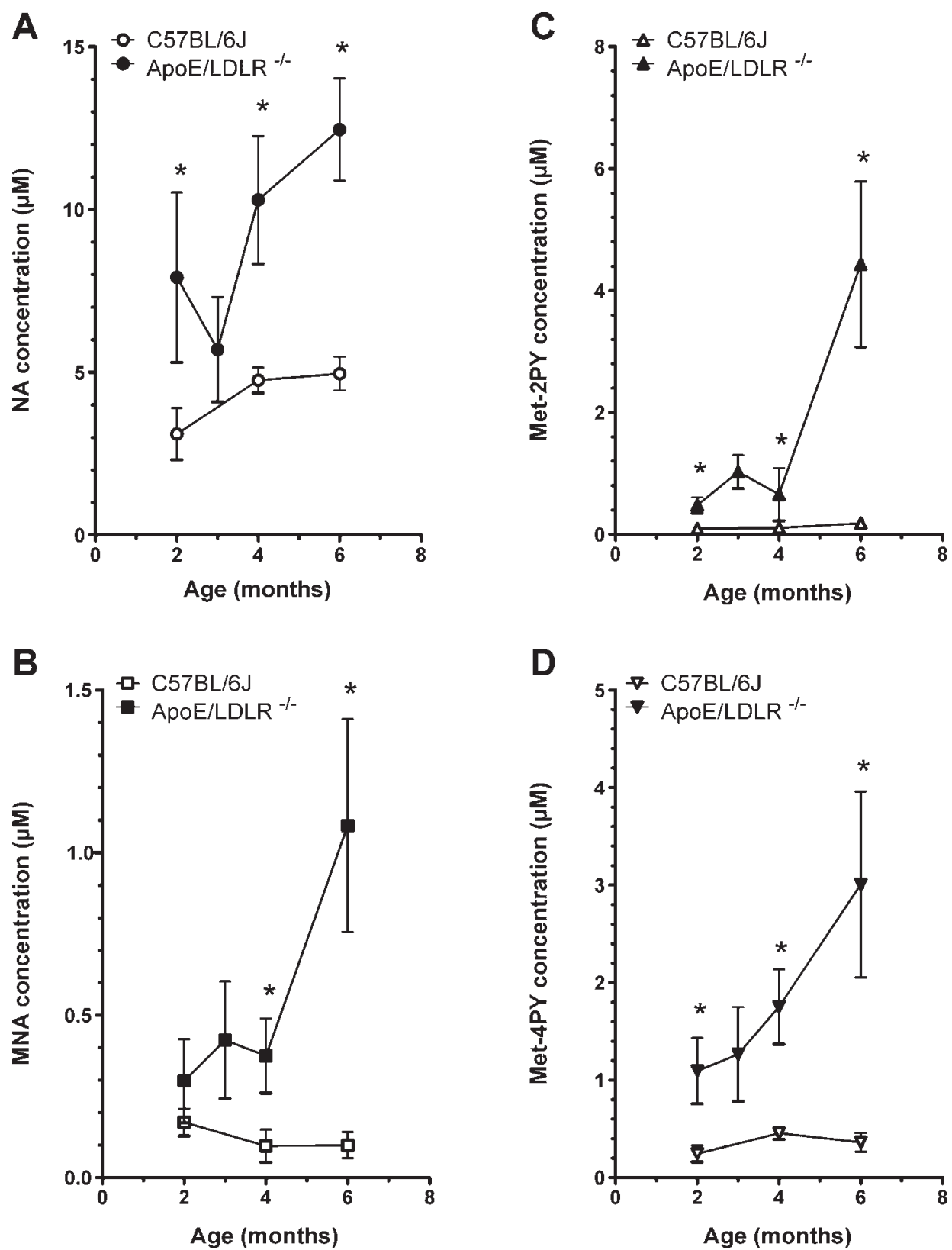

Fig. 4. Increased plasma concentrations of endogenous NA (A), MNA (B), and MNA metabolites Met-2PY (C) and Met-4PY (D) along the progression of atherosclerosis in apoE/LDLR ${ }^{-/-}$mice. Results are presented as the mean $\pm \operatorname{SD}(n=3-5$, * denotes $p<0.05$ Vs. age-matched C57BL/6J mice)

was not solely associated with CD68-positive macrophages. Therefore, the increased activity of MMP-2/ MMP-9 may reflect inflammatory activation of various cell types within atherosclerotic plaques, as well as the deposition of enzymes in the extracellular matrix. As such, the assessment of gelatinase activity provides a relatively easy and comprehensive marker of vascular inflammation in atherosclerotic vessels. This notion stays in line with the important role of
MMP-2 and MMP-9 in the initiation and progression of high cholesterol-driven vascular inflammation and atherosclerosis [11].

Here, we confirmed that the development of atherosclerotic plaques in the aortic roots of apoE/LDLR ${ }^{-/}$ mice could be detected in 3-month-old animals; but not in 2-month-old apoE/LDLR ${ }^{--}$mice [21]. Indeed, 2-month-old apoE/LDLR ${ }^{-1}$ mice displayed no lipid deposition (as assessed by Oil Red-O) or sub- 
endothelial macrophage accumulation (CD68 staining), and negligible gelatinase activity (assessed by in situ zymography). However, NNMT activity and MNA plasma concentrations were elevated during the early stage of atherosclerotic development. Additionally, NO-dependent endothelial function in the aorta was impaired as early as 2 months, which was associated with ultrastructural signs of endothelial activation and hypertrophy [12]. Thus, early activation of an NNMT-dependent pathway for MNA formation coincided with the development of endothelial dysfunction in aorta, which occurred before the formation of atherosclerotic plaques in this model. Subsequently, hepatic NNMT activity and MNA plasma concentrations increased in association with the development of vascular inflammation in the aorta.

The increase in MNA plasma concentration was associated with the significant elevations in NA plasma concentrations, as well as MNA metabolites, such as Met-2PY and Met-4PY. Accordingly, the upregulation of NNMT activity may be linked to concomitant changes in the activity of NA-generating enzymes and/or to the increased catabolism of $\mathrm{NAD}^{+}$[7], as well as changes in the activity of aldehyde oxidase, which catalyzes MNA degradation. Additionally, elevations in plasma concentrations of MNA and Met2PY/Met-4PY in particular may depend on renal function, as these metabolites are renally excreted. Nevertheless, our results suggest a correlation between the development of vascular inflammation in atherosclerosis and MNA formation by NNMT in the liver.

Interestingly, it was recently proposed that enhanced NNMT activity induced by barbiturates may deplete pyridine nucleotides $\left(\mathrm{NAD}^{+}, \mathrm{NADH}\right.$, $\left.\mathrm{NADP}^{+}, \mathrm{NADPH}\right)$ and attenuate pyridine nucleotidedependent protective systems, including NADPHdependent GSH peroxidase and GSSG reductase [16]. However, this seems unlikely to occur in the livers of atherosclerotic mice. Although the pyridine nucleotide content of the liver was not measured in the present work, it was found that GSH peroxidase activity was increased rather than decreased in the livers of apoE/LDLR ${ }^{-/}$mice, while GSSG reductase activity was unchanged (data not shown).

Previous studies have linked the biological activity of NNMT to hyperhomocysteinemia [32]. It is known that SAH and SAM are important intermediates in the synthesis of homocysteine. Although we did not measure plasma homocysteine concentrations, it is unlikely that plasma homocysteine levels of apoE/LDLR ${ }^{-/-}$ mice fed chow (but not methionine-rich) diet [43] would be altered, despite the upregulation in NNMT activity.

Previous studies have also linked the increased NNMT activity to the pathogenesis of Parkinson's disease [29] and tumor growth, migration, and metastasis $[38,46]$. Based on the results of this study, we believe that elevated NNMT activity in the liver and other tissues may not be detrimental but that it may promote a compensatory anti-atherosclerotic response. This may be supported by the profile of exogenous MNA's pharmacological activity, including antithrombotic and anti-inflammatory effects mediated by the $\mathrm{COX}-2 / \mathrm{PGI}_{2}$ pathway $[8,10]$. MNA was also shown to reverse endothelial dysfunction and to affect lipid metabolism, but the involvement of the $\mathrm{COX}-2 / \mathrm{PGI}_{2}$ pathway was not examined in this regard [5].

It is obvious that MNA concentrations in MNAtreated animals [5] exceed endogenous MNA plasma concentrations in this study. Still considering the well-known vasoprotective and anti-atherosclerotic properties of the $\mathrm{COX}-2 / \mathrm{PGI}_{2}$ pathway $[18,19]$ and increased vascular $\mathrm{PGI}_{2}$ synthesis in apoE $\mathrm{LDLR}^{-/}$ mice [12], it is tempting to speculate that NNMTderived MNA may activate vascular $\mathrm{COX}-2 / \mathrm{PGI}_{2}$ pathway in atherosclerosis.

We did not investigate the mechanisms involved in the upregulation of NNMT activity. It is possible that hepatic NNMT is regulated by IL-6 and STAT-3dependent mechanisms [42]. In fact, pro-inflammatory factors released by the activated endothelium or vascular wall $[1,20]$ may provide the stimulus for increased NMMT activity in the liver. If this is true, NNMT activity could represent a novel component of the liver's response to atherosclerosis.

In summary, our study demonstrated that the progression of vascular inflammation and atherosclerosis in apoE/LDLR ${ }^{-/}$mice is associated with increased hepatic NNMT activity, coupled with elevated levels of plasma MNA concentrations. Further studies are necessary to verify whether this response is biologically significant and represents an endogenous antithrombotic and anti-inflammatory pathway in atherosclerosis.

\section{Acknowledgments:}

This work was supported by the Polish Ministry of Science and Higher Education (grant No. N N401015135). We thank Prof. Jerzy Gebicki for encouragement and helpful discussions and Dr. Jan Adamus for providing us with 4-MNA for NMMT assay. 


\section{References:}

1. Aird WC: Endothelium in health and disease. Pharmacol Rep, 2008, 60, 139-143.

2. Aksoy S, Brandriff BF, Ward A, Little PF, Weinshilboum RM: Human nicotinamide N-methyltransferase gene: molecular cloning, structural characterization and chromosomal localization. Genomics, 1995, 29, 555-561.

3. Aksoy S, Szumlanski CL, Weinshilboum RM: Human liver nicotinamide $\mathrm{N}$-methyltransferase. cDNA cloning, expression, and biochemical characterization. J Biol Chem, 1994, 269, 14835-14840.

4. Aoyama K, Matsubara K, Okada K, Fukushima S, Shimizu K, Yamaguchi S, Uezono T et al.: N-methylation ability for azaheterocyclic amines is higher in Parkinson's disease: nicotinamide loading test. J Neural Transm, 2000, 107, 985-995.

5. Bartuś M, Łomnicka M, Kostogrys RB, Kaźmierczak P, Watała C, Słomińska EM, Smoleński RT et al.: 1-Methylnicotinamide (MNA) prevents endothelial dysfunction in hypertriglyceridemic and diabetic rats. Pharmacol Rep, 2008, 60, 127-138.

6. Bathum L, Petersen I, Christiansen L, Konieczna A, Sorensen TI, Kyvik KO: Genetic and environmental influences on plasma homocysteine: results from a Danish twin study. Clin Chem, 2007, 53, 971-979.

7. Bortell R, Moss J, McKenna RC, Rigby MR, Niedzwiecki D, Stevens LA, Patton WA et al.: Nicotinamide adenine dinucleotide (NAD) and its metabolites inhibit T lymphocyte proliferation: role of cell surface NAD glycohydrolase and pyrophosphatase activities. J Immunol, 2001, 167, 2049-2059.

8. Bryniarski K, Biedron R, Jakubowski A, Chlopicki S, Marcinkiewicz J: Anti-inflammatory effect of 1-methylnicotinamide in contact hypersensitivity to oxazolone in mice; involvement of prostacyclin. Eur J Pharmacol, 2008, 578, 332-338.

9. Brzozowski T, Konturek PC, Chlopicki S, Sliwowski Z, Pawlik M, Ptak-Belowska A, Kwiecien S et al.: Therapeutic potential of 1-methylnicotinamide against acute gastric lesions induced by stress: role of endogenous prostacyclin and sensory nerves. J Pharmacol Exp Ther, 2008, 326, 105-116.

10. Chlopicki S, Swies J, Mogielnicki A, Buczko W, Bartus M, Lomnicka M, Adamus J, Gebicki J: 1-Methylnicotinamide (MNA), a primary metabolite of nicotinamide, exerts anti-thrombotic activity mediated by a cyclooxygenase-2/prostacyclin pathway. Br J Pharmacol, 2007, 152, 230-239.

11. Chow AK, Cena J, Schulz R: Acute actions and novel targets of matrix metalloproteinases in the heart and vasculature. Br J Pharmacol, 2007, 152, 189-205.

12. Csanyi G, Franczyk-Zarow F, Gajda M, Mateuszuk L, Gwozdz P, Jawien J, Wojcik L et al.: NO, COX-2/PGI 2 and EDHF pathways in endothelial dysfunction in aorta of apoE/LDLR ${ }^{-1}$ mice; relationship with the development of atherosclerotic plaques and vascular inflammation. Cardiovasc Pathol, 2009, (submitted).
13. Cuomo R, Dattilo M, Pumpo R, Capuano G, Boselli L, Budillon G: Nicotinamide methylation in patients with cirrhosis. J Hepatol, 1994, 20, 138-142.

14. Delaney J, Hodson MP, Thakkar H, Connor SC, Sweatman BC, Kenny SP, McGill PJ et al.: Tryptophan-NAD ${ }^{+}$ pathway metabolites as putative biomarkers and predictors of peroxisome proliferation. Arch Toxicol, 2005, 79, 208-223.

15. Dong H, Toyoda N, Yoneyama H, Kurachi M, Kasahara $\mathrm{T}$, Inadera $\mathrm{H}$, Hashimoto S, Matsushima K: Gene expression profile analysis of the mouse liver during bacteriainduced fulminant hepatitis by a cDNA microarray system. Biochem Biophys Res Commun, 2002, 298, 675-686.

16. Dostalek M, Hardy KD, Milne GL, Morrow JD, Chen C, Gonzales FJ, Gu J et al.: Development of oxidative stress by cytochrome $\mathrm{P} 450$ induction in rodents is selective for barbiturates and related to loss of pyridine nucleotidedependent protective systems. J Biol Chem, 2008, 283, 17147-17157.

17. Galis ZS, Khatri JJ: Matrix metalloproteinases in vascular remodeling and atherogenesis: the good, the bad, and the ugly. Circ Res, 2002, 90, 251-262.

18. Grosser T, Fries S, FitzGerald GA: Biological basis for the cardiovascular consequences of COX-2 inhibition: therapeutic challenges and opportunities. J Clin Invest, 2006, 116, 4-15.

19. Gryglewski RJ: Prostacyclin among prostanoids. Pharmacol Rep, 2008, 60, 3-11.

20. Hansson GK, Robertson AK, Soderberg-Naucler C: Inflammation and atherosclerosis. Annu Rev Pathol, 2006, 1, 297-329.

21. Ishibashi S, Herz J, Maeda N, Goldstein JL, Brown MS: The two-receptor model of lipoprotein clearance: tests of the hypothesis in "knockout" mice lacking the low density lipoprotein receptor, apolipoprotein E, or both proteins. Proc Natl Acad Sci USA, 1994, 91, 4431-4435.

22. Jang JS, Cho HY, Lee YJ, Ha WS, Kim HW: The differential proteome profile of stomach cancer: identification of the biomarker candidates. Oncol Res, 2004, 14, 491-499.

23. Jawien J, Csanyi G, Gajda M, Mateuszuk L, Lomnicka L, Korbut R, Chlopicki S: Ticlopidine attenuates progression of atherosclerosis in apolipoprotein $\mathrm{E}$ and low density lipoprotein receptor double knockout mice. Eur J Pharmacol, 2007, 556, 129-135.

24. Kassem HS, Sangar V, Cowan R, Clarke N, Margison GP: A potential role of heat shock proteins and nicotinamide N-methyl transferase in predicting response to radiation in bladder cancer. Int J Cancer, 2002, 101, 454-460.

25. Katsuda S, Kaji T: Atherosclerosis and extracellular matrix. J Atheroscler Thromb, 2003, 10, 267-274.

26. Kurpius MP, Alexander B: Rates of in vivo methylation of desipramine and nortriptyline. Pharmacotherapy, 2006, 26, 505-510.

27. Li K, Prow T, Lemon SM, Beard MR: Cellular response to conditional expression of hepatitis $\mathrm{C}$ virus core protein in Huh7 cultured human hepatoma cells. Hepatology, 2002, 35, 1237-1246. 
28. Maiese K, Chong ZZ: Nicotinamide: necessary nutrient emerges as a novel cytoprotectant for the brain. Trends Pharmacol Sci, 2003, 24, 228-232.

29. Matsubara K, Aoyama K, Suno M, Awaya T: N-methylation underlying Parkinson's disease. Neurotoxicol Teratol, 2002, 24, 593-598.

30. Ohkubo M, Sakiyama S, Fujimura S: Increase of nicotinamide methyltransferase and N1-methyl-nicotinamide oxidase activities in the livers of the rats administered alkylating agents. Cancer Lett, 1983, 21, 175-181.

31. Pumpo R, Sarnelli G, Spinella A, Budillon G, Cuomo R: The metabolism of nicotinamide in human liver cirrhosis: a study on N-methylnicotinamide and 2-pyridone-5carboxamide production. Am J Gastroenterol, 2001, 96, 1183-1187.

32. Riederer M, Erwa W, Zimmermann R, Frank S, Zechner $\mathrm{R}$ : Adipose tissue as a source of nicotinamide N-methyltransferase and homocysteine. Atherosclerosis, 2008, in press.

33. Rini J, Szumlanski C, Guerciolini R, Weinshilboum RM: Human liver nicotinamide N-methyltransferase: ionpairing radiochemical assay, biochemical properties, and individual variation. Clin Chim Acta, 1990, 186, 359-374.

34. Rivera S, Ogier C, Jourquin J, Timsit S, Szklarczyk AW, Miller K, Gearing AJ et al.: Gelatinase B and TIMP-1 are regulated in a cell- and time-dependent manner in association with neuronal death and glial reactivity after global forebrain ischemia. Eur J Neurosci, 2002, 15, 19-32.

35. Roessler M, Rollinger W, Palme S, Hagmann ML, Berndt $\mathrm{P}$, Engel AM, Schneidinger B et al.: Identification of nicotinamide $\mathrm{N}$-methyltransferase as a novel serum tumor marker for colorectal cancer. Clin Cancer Res, 2005, 11, 6550-6557.

36. Sano A, Takimoto N, Takitani S: Fluorometric assay of nicotinamide methyltransferase with a new substrate, 4-methylnicotinamide. Chem Pharm Bull (Tokyo), 1989, 37, 3330-3332.

37. Sartini D, Muzzonigro G, Milanese G, Pierella F, Rossi V, Emanuelli M: Identification of nicotinamide N-methyltransferase as a novel tumor marker for renal clear cell carcinoma. J Urol, 2006, 176, 2248-2254.

38. Sartini D, Santarelli A, Rossi V, Goteri G, Rubini C, Ciavarello C, Lo Muzio L, Emanuelli M: Nicotinamide $\mathrm{N}$-methyltransferase upregulation inversely correlates with lymph node metastasis in oral squamous cell carcinoma. Mol Med, 2007, 13, 415-421.

39. Seifert R, Hoshino J, Kroger H: Nicotinamide methylation. Tissue distribution, developmental and neoplastic changes. Biochim Biophys Acta, 1984, 801, 259-264.
40. Slominska EM, Adamski P, Lipinski M, Swierczynski J, Smolenski RT: Liquid chromatographic/mass spectrometric procedure for measurement of NAD catabolites in human and rat plasma and urine. Nucleosides Nucleotides Nucleic Acids, 2006, 25, 1245-1249.

41. Souto JC, Blanco-Vaca F, Soria JM, Buil A, Almasy L, Ordoñez-Llanos J, Martin-Campos JL et al.: A genomewide exploration suggests a new candidate gene at chromosome 11q23 as the major determinant of plasma homocysteine levels: results from the GAIT project. Am J Hum Genet, 2005, 76, 925-933.

42. Tomida M, Ohtake H, Yokota T, Kobayashi Y, Kurosumi M: Stat3 up-regulates expression of nicotinamide $\mathrm{N}$-methyltransferase in human cancer cells. J Cancer Res Clin Oncol, 2008, 134, 551-559.

43. Troen AM, Lutgens E, Smith DE, Rosenberg IH, Selhub $\mathrm{J}$ : The atherogenic effect of excess methionine intake. Proc Natl Acad Sci USA, 2003, 100, 15089-15094.

44. van Driel LM, Smedts HP, Helbing WA, Isaacs A, Lindemans J, Litterlinden AG, van Duijn CM et al.: Eight-fold increased risk for congenital heart defects in children carrying the nicotinamide $\mathrm{N}$-methyltransferase polymorphism and exposed to medicines and low nicotinamide. Eur Heart J, 2008, 29, 1424-1431.

45. Weinshilboum R: Methyltransferase pharmacogenetics. Pharmacol Ther, 1989, 43, 77-90.

46. Wu Y, Siadaty MS, Berens ME, Hampton GM, Theodorescu D: Overlapping gene expression profiles of cell migration and tumor invasion in human bladder cancer identify metallothionein $1 \mathrm{E}$ and nicotinamide N-methyltransferase as novel regulators of cell migration. Oncogene, 2008, 27, 6679-6689.

47. Xu J, Capezzone M, Xu X, Hershman JM: Activation of nicotinamide $\mathrm{N}$-methyltransferase gene promoter by hepatocyte nuclear factor-1beta in human papillary thyroid cancer cells. Mol Endocrinol, 2005, 19, 527-539.

48. Yan L, Otterness DM, Craddock TL, Weinshilboum RM: Mouse liver nicotinamide N-methyltransferase: cDNA cloning, expression, and nucleotide sequence polymorphisms. Biochem Pharmacol, 1997, 54, 1139-1149.

49. Yao M, Tabuchi H, Nagashima Y, Baba M, Nakaigawa $\mathrm{N}$, Ishiguro $\mathrm{H}$, Hamada $\mathrm{K}$ et al.: Gene expression analysis of renal carcinoma: adipose differentiation-related protein as a potential diagnostic and prognostic biomarker for clear-cell renal carcinoma. J Pathol, 2005, 205, 377-387.

Received:

December 16, 2008; in revised form: January 26, 2009. 